\title{
Factores de riesgo para alteraciones de la densidad mineral ósea en mujeres posmenopáusicas
}

José René Guerra R. ${ }^{1}$, José Ramón Urdaneta M., $P h D^{2}$, Noren Villalobos I., $P h D^{1,3}$, Alfi Contreras Benítez. ', José García I., $P h D^{1,5}$, Nasser Saleh Baabel Z., PhD ${ }^{5}$, Mery Guerra V., PhD ${ }^{5}$, Marielis Lozada ${ }^{1}{ }^{1}$

${ }^{1}$ Programa de Especialización en Obstetricia y Ginecología. Facultad de Medicina. ${ }^{2}$ Cátedra de Anatomía, Escuela de Bioanálisis, Facultad de Medicina. ${ }^{3}$ Cátedra de Anatomía, Facultad de Medicina. ${ }^{4}$ Cátedra de Anatomía Humana, Facultad de Odontología. ${ }^{5}$ Departamento de Obstetricia y Ginecología, Facultad de Medicina. Universidad del Zulia. Venezuela.

\section{RESUMEN}

Objetivo: Determinar los factores de riesgo para presentar alteraciones de la densidad mineral ósea (DMO) en mujeres posmenopáusicas atendidas en la consulta de Menopausia y Climaterio de la Maternidad "Dr. Armando Castillo Plaza", de Maracaibo, Venezuela. Métodos: Investigación descriptiva, con diseño no experimental y transeccional, donde se evaluó la DMO y los factores de riesgo para presentar osteoporosis en 60 mujeres posmenopáusicas. Resultados: La medición de la DMO resultó en promedio de 957,45 \pm 149,95 y 905,00 $\pm 151,25$ gramos, con índices T de -0,52 \pm 1,66 y -0,55 $\pm 2,67$ en columna vertebral y cuello femoral, respectivamente. Se estableció una prevalencia de osteoporosis del $10 \%$ y $5 \%$, y de osteopenia del $43,3 \%$ y $50 \%$ en columna vertebral y fémur, respectivamente. La menopausia quirúrgica (OR $[95 \% \mathrm{Cl}]=4,75[1,58-14,25] ; p=0,004)$, el consumo excesivo de café $(\mathrm{OR}[95 \% \mathrm{Cl}]=3,20[1,40-7,101]$; $p=0,000)$ o gaseosas (OR $[95 \% \mathrm{Cl}]=2,50[1,18-5,60] ; p=0,002)$, tabaquismo (OR $[95 \% \mathrm{Cl}]=1,70[1,10-$ $2,80] ; p=0,013)$ y la ausencia de suplementación de calcio más vitamina $D(O R[95 \% \mathrm{Cl}]=1,70[1,00-2,80]$; $p=0,019)$ resultaron ser factores significativamente asociados al diagnóstico de osteoporosis u osteopenia $(p<0,05)$. Conclusión: Las mujeres posmenopáusicas evaluadas presentan una alta prevalencia de alteraciones en la DMO, principalmente osteopenia, y factores de riesgo para presentar Osteoporosis.

\section{PALABRAS CLAVE: Densidad mineral ósea, factores de riesgo, menopausia, osteopenia, osteoporosis}

\section{SUMMARY}

Aim: To determine risk factors for presenting alterations in bone mineral density (BMD) in postmenopausal women attending the consultation of Menopause and Menopause Maternity "Dr. Armando Castillo Plaza", Maracaibo, Venezuela. Methods: A descriptive research with non-experimental and transactional design where was evaluated BMD and risk factors for developing osteoporosis in 60 postmenopausal women. Results: The BMD measurement was averaged in $957.45 \pm 149.95$ and $905.00 \pm 151.25$ grams; with T indexes of $-0.52 \pm 1.66$ and $-0.55 \pm 2.67$ in spine and femoral neck, respectively. The prevalence of osteoporosis was $10 \%$ and $5 \%$, while osteopenia was $43.3 \%$ and $50 \%$ in spine and femur, respectively. Surgical menopause $(\mathrm{OR}[95 \% \mathrm{Cl}]=4.75[1.58$ to 14.25$] ; p=0.004)$; excessive coffee consumption $(\mathrm{OR}[95 \% \mathrm{Cl}]=3.20$ $[1,40-7.101] ; p=0.000)$ or gaseous beverages (OR $[95 \% \mathrm{Cl}]=2.50$ [1.18 to 5.60$] ; p=0.002)$; smoking $(\mathrm{OR}$ 
[95\% $\mathrm{Cl}]=1.70$ [1.10 to 2.80$] ; \mathrm{p}=0.013)$ and the absence of supplemental calcium plus vitamin $\mathrm{D}(\mathrm{OR}$ [95\% $\mathrm{Cl}]=1.70$ [1.00 to 2.80]; $p=0.019$ ) were be factors significantly associated with the diagnosis of osteoporosis or osteopenia $(p<0.05)$. Conclusion: The evaluated postmenopausal women have a high prevalence of abnormal BMD, especially osteopenia, and risk factors for developing osteoporosis.

\section{KEY WORDS: Bone mineral density, risk factors, menopause, osteoporosis, osteopenia}

\section{INTRODUCCIÓN}

La osteoporosis es un problema de salud pública emergente dado el envejecimiento progresivo de la sociedad y a los efectos devastadores de las fracturas osteoporóticas, que aumentan significativamente la morbimortalidad y alteran la funcionalidad de los pacientes afectados $(1,2)$, estimándose que alrededor 75 millones de personas tan sólo en los Estados Unidos, Europa y Japón la padecen (3). En los Estados Unidos aproximadamente 8 millones de mujeres presentan ésta patología (4), reportándose en este país que $30 \%$ y $54 \%$ de las mujeres caucásicas posmenopáusicas presentan osteoporosis u osteopenia, respectivamente, a nivel de cadera, columna lumbar o del radio (3).

La Organización Mundial de la Salud (OMS) considera la presencia de osteoporosis cuando la densidad mineral ósea fue menor de 2,5 desviaciones estándar (DE) del pico normal de masa ósea (5), debiendo ser diferenciada del término osteopenia, el cual es más específico y descriptivo, haciendo referencia simplemente a la rarefacción del esqueleto, visto en las radiografías y es más evidente tanto en el esqueleto axial como en los extremos de los huesos largos, donde el hueso trabecular es abundante (3).

Aunque para llegar al pico de masa ósea existe una clara predisposición genética, tanto el pico como la pérdida posterior pueden ser modificados por diversos factores de riesgos que pueden reducir o incrementar la densidad mineral ósea (DMO) (6), la identificación de estos factores y su abordaje precoz constituyen una estrategia a seguir para reducir la incidencia de fracturas osteoporóticas $(1,7)$. Se consideran como factores no modificables la edad mayor a 65 años (8), raza caucásica o asiática $(9,10)$, genética $(11,12)$, antecedentes de fractura osteoporótica en familiares de primer grado de consanguineidad e historia familiar de osteoporosis $(9,12)$, menopausia precoz (13-15), menopausia quirúrgica (16-18), más de 10 años de evolución de la menopausia $(19,20)$, presentar demencia o fractura en la tercera edad (3).

Por su parte, un índice de masa corporal $<19$ $\mathrm{kg} / \mathrm{m} 2$ (3), déficit ingesta de calcio $(<1.000-1.200$ $\mathrm{mg} /$ día) $(21,22)$, déficit de ingesta de vitamina $D$
$<400-800$ U/día (9), tabaquismo $(23,24)$, ingesta excesiva de alcohol (22), hábito caféico (25), consumo excesivo de bebidas gaseosas (26), ejercicio físico intenso o sedentarismo (19), inmovilización (25), exceso de proteínas en la dieta o dieta vegetariana $(6,27)$, representan factores de riesgo modificables. La presencia de uno o dos factores de riesgo y DMO baja aumenta casi 3 veces $(R R=$ $2,6)$ la probabilidad de fractura de cadera, mientras que en presencia de 5 o más factores de riesgo la aumenta a 27,3 veces (28).

El objetivo de este estudio es determinar los factores de riesgo para presentar alteraciones en la DMO en mujeres posmenopáusicas atendidas en la Maternidad "Dr. Armando Castillo Plaza", de Maracaibo, Venezuela.

\section{PACIENTES Y MÉTODOS}

Investigación descriptiva con diseño no experimental y transeccional, realizado en una población de mujeres posmenopáusicas atendidas en la consulta de Menopausia y Climaterio, de la cual se tomó una muestra intencionada de 60 mujeres mayores a 40 años de edad, con diagnóstico de menopausia (FSH mayor a $40 \mathrm{mlU} / \mathrm{L}$ y/o 12 meses de amenorrea), sin recibir o haber sido tratadas con terapia de reemplazo hormonal. Se excluyeron pacientes con enfermedades con conocida repercusión ósea, tales como diabetes, hiperparatiroidismo, litiasis renal, enfermedad pulmonar obstructiva crónica, enfermedades reumatológicas, hipertiroidismo, gastrectomía, síndrome de malabsorción, resección intestinal, enfermedades crónicas hepáticas y renales. Asimismo, fueron excluidas pacientes bajo tratamiento con anticoagulantes (heparina), anticonvulsantes (fenitoína, fenobarbital), ciclosporina, tacrolimus, quimioterapia con citotóxicos, glucocorticoides, hormona adrenocorticotrópica, agonistas de la hormona liberadora de gonadotropina, metotrexate, litio, tiroxina, o nutrición parenteral.

Las pacientes participaron voluntariamente y sin obtener ningún beneficio económico y/o material, asimismo, previa explicación del procedimiento a seguir, les fue solicitado su consentimiento informado por escrito. El estudio propuesto no representó riesgo para las pacientes involucradas ni se 
vulneraron las normas éticas de la declaración del Helsinki, siendo el protocolo de estudio previamente aprobado por el Comité de Bioética de la institución ámbito del estudio.

Se realizó una consulta ginecológica, en la cual por medio de la entrevista clínica se registraron en una ficha de trabajo los siguientes datos: edad cronológica, edad de la menopausia y menarquía, tiempo de la menopausia, nivel educativo, estado civil, nivel socioeconómico, e ingreso familiar, además de los diversos factores de riesgo a constatar en estas pacientes. Con relación a los factores de riesgo, en la evaluación de los antecedentes de fracturas personales y familiares se tomaron las fracturas probablemente osteoporóticas, ocurridas después de los 45 años de edad, por traumas mínimos, comprometiendo las muñecas, el húmero, el fémur o la tibia, descartándose los producidos por accidentes automovilísticos, asimismo, se consideró como antecedente familiar de osteoporosis si hubo reemplazo de cadera por fractura, cifosis marcada o diagnóstico mediante DEXA (Dual-energy X-ray absorptiometry) en familiares de primer grado.

Se evaluó en la historia ginecológica la edad de la menopausia para establecer la presencia de menopausia precoz o anticipada, cuando ocurra antes de los 40 años, así como el tiempo transcurrido desde la menopausia, considerándose como factor de riesgo una evolución superior a 10 años. De igual manera, para establecer el tipo de menopausia se investigó el antecedente de histerectomía, la edad a la que fue realizada y si hubo o no preservación de los ovarios.

Respecto al tabaquismo, se clasificó como fumadora a la persona que regularmente consumía por lo menos un cigarrillo diario y durante el último año como mínimo; ex fumadora aquella que no había fumado durante el último año, pero confesaba haber consumido cigarrillos entre uno y diez años antes de la encuesta; no fumadora a las que nunca habían fumado o habían dejado ese hábito por lo menos diez años anterior al estudio y como fumadora pasiva a aquellas mujeres que no son fumadoras activas pero que pasaban más de 8 horas diarias en ambientes cerrados donde se consuma tabaco.

En cuanto a la actividad física se consideraron como sedentarias, a aquellas mujeres que nunca realizaban ejercicio físico, las que lo practicaban menos de 3 veces por semana, o las que perdían menos de $1.500 \mathrm{kcal} /$ día debido a su actividad física. Se catalogó un consumo excesivo de café una frecuencia diaria de más de 12 tazas ( 1 taza $=50$ $\mathrm{ml}$ ), equivalente a más de $300 \mathrm{ml}$ diarios. El consumo de gaseosas se midió por el número de vasos de 8 onzas $(200 \mathrm{ml})$ consumidos al día, considerándose una ingesta elevada de fósforo cuando era superior a 1,2 g/día. En relación al consumo de alcohol se estableció como factor de riesgo el consumo de más de $10 \mathrm{ml}$ diarios, tres veces o más por semana.

En cuanto a factores nutricionales, como el tipo de dieta, se consideraron aquellas con alto consumo de proteínas y las dietas vegetarianas. Respecto al consumo de calcio dietario se consideró como inadecuado una ingesta de calcio inferior a 1,2 gramos, equivalentes a consumir menos de 500 $\mathrm{ml}$ diarios de leche o a consumir menos de 3 veces por semanas otras fuentes de calcio como yogurt o quesos frescos, mientras que para la vitamina $D$ se consideró inadecuada la ingesta de menos de $400 \mathrm{mg} /$ día, con una exposición solar menor de 15 minutos diarios. También fue indagado el consumo regular o no de suplementos de calcio y vitamina $D$ que aporten 1500 y 400 mg diarios, respectivamente. Por último, el índice de masa corporal (IMC), se analizó de acuerdo con los criterios de la Organización Mundial de la Salud (29), considerándose que hay un déficit si el IMC es igual o menor de $19 \mathrm{~kg} /$ $\mathrm{m}^{2}$, normal entre 20 y $24,9 \mathrm{~kg} / \mathrm{m}^{2}$, sobrepeso entre 25 y $29,9 \mathrm{~kg} / \mathrm{m} 2$ u obesidad si es mayor de $30 \mathrm{~kg} /$ $\mathrm{m}^{2}$.

Se realizó la medición de la DMO de columna lumbar y cuello de fémur, procedimientos efectuados en el Departamento de Radiología e Imágenes Diagnósticas de la institución por un mismo evaluador, por medio de un densitómetro Hologic QDR 4500 DXA (Hologic, Inc., Bedford, Mass) en columna lumbar (L2-L4) y en cuello de fémur. La técnica se basa en la atenuación por el cuerpo del paciente, de un haz de radiación generado por una fuente de rayos $X$ con dos niveles de potencia, siendo los coeficientes de variación durante las pruebas de menos de $2 \%$. Los valores obtenidos fueron expresados en $\mathrm{g} / \mathrm{cm}^{2}$, y se utilizó además el Índice T expresado en desviaciones estándar (DE), para clasificar los resultados obtenidos como normal (> $\mathrm{T}-1,0 \mathrm{DE})$, osteopenia (T-1,0 y T-2,4 DE) u osteoporosis ( $\geq \mathrm{T}-2,5 \mathrm{DE})$, de acuerdo con los criterios propuestos por la OMS (5).

Los datos obtenidos se procesaron mediante el Paquete Estadístico para Ciencias Sociales (SPSS, versión 19), ejecutándose un análisis estadístico descriptivo, donde los datos se expresaron mediante frecuencias absolutas o relativas (porcentajes), medidas de tendencia central (medias) y de dispersión (rango y DE). La estimación del riesgo según los factores investigados, se realizó mediante un análisis de regresión logística, expresando el riesgo en términos de Odds Ratios (OR) con sus respectivos intervalos de confianza del 95\% (IC95\%) determinándose su significancia mediante la prueba del Chi cuadrado o por el test exacto de Fischer cuando la frecuencia en una casilla era menor a 5; ambas con significancia de $p<0,05$. 


\section{RESULTADOS}

En la Tabla I se describen las principales características de las pacientes posmenopáusicas estudiadas, encontrándose que se trataban de mujeres de edad mediana con una edad promedio al momento de la entrevista de 53 años, de baja talla, con sobrepeso y un promedio de 8 años posterior a la menopausia.

\section{Tabla I}

CARACTERIZACIÓN DE LA MUESTRA DE MUJERES POSMENOPÁUSICAS

\begin{tabular}{lr}
\hline Variables & \multicolumn{1}{c}{$\overline{\mathrm{X}} \pm \mathrm{DE}$} \\
\hline Edad (años) & $53,53 \pm 7,66$ \\
Menarquía (años) & $11,83 \pm 1,41$ \\
Edad de Menopausia (años) & $44,84 \pm 3,91$ \\
Tiempo de posmenopausia (años) & $8,96 \pm 5,81$ \\
Peso (kg) & $63,96 \pm 8,62$ \\
Talla (m) & $1,52 \pm 0,05$ \\
IMC (kg/m & $26,75 \pm 2,89$
\end{tabular}

$\overline{\mathrm{X}} \pm \mathrm{DE}:$ Promedio \pm Desviación Estándar

En la Tabla II, se presentan los valores medios de la DMO y los índices T, para columna vertebral y fémur derecho, presentándose los promedios de estos índices dentro del rango normal. Sin embargo, al establecer las alteraciones en la DMO de mujeres posmenopáusicas (Tabla III), se evidenció que a nivel de columna vertebral se observó que $53,3 \%$ presentaron un diagnóstico alterado, bien sea osteopenia $(43,3 \% ; n=26)$ u osteoporosis $(10 \% ; n=6)$; mientras que a nivel del cuello femoral 33 mujeres (55\%) presentaron una DMO alterada (50\% osteopenia y $5 \%$ osteoporosis).

\section{Tabla II \\ DENSIDAD MINERAL ÓSEA EN COLUMNA LUMBAR Y CUELLO FEMORAL DE MUJERES POSMENOPÁUSICAS}

\begin{tabular}{lc}
\hline Variables & $\overline{\mathrm{X}} \pm \mathrm{DE}$ \\
\hline Densidad mineral ósea: & \\
- Columna lumbar & $957,45 \pm 149,95$ \\
- Fémur derecho & $905,00 \pm 151,25$ \\
Índice T: & \\
- Columna lumbar & $-0,52 \pm 1,66$ \\
- Fémur derecho & $-0,55 \pm 2,67$ \\
\hline
\end{tabular}

$\overline{\mathrm{X}} \pm \mathrm{DE}$ : Promedio \pm Desviación Estándar.
Tabla III

ALTERACIONES DE LA DENSIDAD MINERAL ÓSEA DE MUJERES POSMENOPÁUSICAS

\begin{tabular}{lcc}
\hline Diagnóstico & $\mathrm{n}$ & $\%$ \\
\hline Columna vertebral: & & \\
Normal & 28 & 46,67 \\
Alterada* & 32 & 53,33 \\
- Osteopenia & 26 & 43,33 \\
- Osteoporosis & 6 & 10,00 \\
Fémur: & & \\
Normal & 27 & 45,00 \\
Alterada* & 33 & 55,00 \\
- Osteopenia & 30 & 50,00 \\
- Osteoporosis & 3 & 05,00 \\
\hline
\end{tabular}

* Osteopenia-Osteoporosis. $n=60$

La Tabla IV muestra los factores de riesgos no modificables para presentar alguna alteración de la $\mathrm{DMO}$, siendo la menopausia quirúrgica el único factor de riesgo asociado al diagnóstico de DMO alterada, la cual demostró tener un riesgo significativo de casi 5 veces más probabilidad de presentar osteopenia u osteoporosis (OR [IC95\%]= 4,75 [1,58-14,25]; $p<0,05)$.

En cuanto a los factores de riesgo modificables (Tabla V), se evidenció que los principales factores asociados al diagnóstico de osteoporosis u osteopenia, en orden de importancia, resultaron ser el consumo excesivo de café (OR [IC95\%]= 3,2 [1,4$7,1] ; p<0,05)$ o de bebidas gaseosas (OR [IC95\%]= $2,5[1,18-5,6] ; p<0,05)$; mientras que el hábito tabáquico (OR $[$ IC95\%]= $1,7[1,1-2,8] ; p<0,05)$ y la ausencia de suplementación de calcio más vitamina $D(O R[I C 95 \%]=1,7[1,0-2,8] ; p<0,05)$ a pesar de mostrar diferencias causales estadísticamente significativas, la intensidad de la asociación establecida fue débil $(O R<2)$.

\section{DISCUSIÓN}

La investigación efectuada determinó un gran número de mujeres posmenopáusicas con factores de riesgo para presentar osteoporosis, encontrándose una prevalencia de osteopenia de $43,3 \%$ y $50 \%$, a nivel vertebral y del cuello femoral, respectivamente, mientras que la osteoporosis se encontró en $10 \%$ y $5 \%$ en dichas mediciones. Estas cifras, se aproximan a la prevalencia de osteoporosis vertebral del $12,1 \%-17,6 \%$ y del $7,9 \%-22 \%$ para cuello femoral aportada por investigaciones realizadas en Latinoamérica (29), así como el 45,9\% de osteopenia reportada en mujeres cubanas (30). 


\section{Tabla IV \\ FACTORES DE RIESGO NO MODIFICABLES PARA OSTEOPOROSIS EN MUJERES POSMENOPÁUSICAS}

\begin{tabular}{|c|c|c|c|c|c|c|c|}
\hline \multirow{3}{*}{$\begin{array}{l}\text { FACTOR } \\
\text { DE RIESGO }\end{array}$} & \multicolumn{4}{|c|}{$\mathrm{DMO}$} & \multirow{3}{*}{ OR } & \multirow{3}{*}{ IC95\% } & \multirow{3}{*}{ Valor $p$} \\
\hline & \multicolumn{2}{|c|}{ NORMAL } & \multicolumn{2}{|c|}{ ALTERADA* } & & & \\
\hline & $\mathrm{n}$ & $\%$ & $\mathrm{n}$ & $\%$ & & & \\
\hline Edad ** & & & & & 1,3 & $0,40-3,60$ & 0,580 \\
\hline$\leq 53$ años & 15 & 25,0 & 16 & 26,7 & & & \\
\hline$\geq 54$ años & 12 & 20,0 & 17 & 28,3 & & & \\
\hline Raza & & & & & 0,9 & $0,48-1,90$ & 0,903 \\
\hline Caucásica & 24 & 40,0 & 29 & 48,3 & & & \\
\hline Otra & 3 & 5,0 & 4 & 6,7 & & & \\
\hline Menopausia precoz ( $\leq 40$ años) & & & & & 1,12 & $0,68-1,80$ & 0,650 \\
\hline Presente & 6 & 10,0 & 9 & 15,0 & & & \\
\hline Ausente & 21 & 35,0 & 24 & 40,0 & & & \\
\hline Tipo de menopausia & & & & & 4,75 & $1,58-14,25$ & 0,004 \\
\hline Natural & 19 & 31,7 & 11 & 18,3 & & & \\
\hline Quirúrgica & 11 & 18,3 & 22 & 36,6 & & & \\
\hline Tiempo de menopausia & & & & & 1,16 & $0,71-1,80$ & 0,520 \\
\hline$<10$ años & 15 & 25,0 & 21 & 35,0 & & & \\
\hline$\geq 10$ años & 12 & 20,0 & 12 & 20,0 & & & \\
\hline Historia familiar de osteoporosis & & & & & 1,68 & $1,10-1,15$ & 0,116 \\
\hline Presente & 1 & 1,7 & 6 & 10,0 & & & \\
\hline Ausente & 26 & 43,3 & 27 & 45,0 & & & \\
\hline $\begin{array}{l}\text { Historia familiar de fracturas } \\
\text { osteoporóticas }\end{array}$ & & & & & 1,23 & $0,07-20,64$ & 0,885 \\
\hline Presente & 1 & 1,7 & 1 & 1,7 & & & \\
\hline Ausente & 26 & 43,3 & 32 & 53,3 & & & \\
\hline Antecedentes de fracturas & & & & & 1,23 & $0,07-20,64$ & 0,885 \\
\hline Presente & 1 & 1,7 & 1 & 1,7 & & & \\
\hline Ausente & 26 & 43,3 & 32 & 53,3 & & & \\
\hline
\end{tabular}

* Osteopenia-Osteoporosis. DMO: Densidad mineral ósea.

** Se toma p50 como punto de corte; al no contar en la muestra con sujetos $\geq 65$ años.

Sin embargo, otra investigación en mujeres zulianas reportó una prevalencia mayor, tanto de osteoporosis como de osteopenia, $62 \%$ y $38 \%$ respectivamente (31). Asimismo, los resultados presentados son menores tanto a los encontrados en mujeres caucásicas posmenopáusicas norteamericanas donde se reportó hasta un $30 \%$ de osteoporosis y $54 \%$ de osteopenia (3), como también, a las prevalencias publicadas en México de 39,8\% y $13,6 \%$ (32) y de $34,5 \%$ y $17,9 \%$ (33) para osteopenia y osteoporosis, respectivamente.

Respecto a los factores no modificables, se demostró que las mujeres que presentaron meno- pausia quirúrgica debido al antecedente de histerectomía con ooforectomía bilateral, presentaban un riesgo significativo de casi 5 veces más probabilidad de presentar alguna alteración en la DMO $(p<0,05)$. Similar hallazgo se reportó en mujeres mexicanas (28), aunque el riesgo encontrado en esta serie fue mucho más elevado. Asimismo, coinciden con otras investigaciones que han reportado que los valores de la DMO en columna lumbar al sexto mes del postoperatorio era significativamente menores en comparación con el período preoperatorio $(p=0,0001)(34)$ y hasta $6,7 \%$ menos luego del año de la ooforectomía bilateral (35). 


\section{Tabla V}

FACTORES DE RIESGO MODIFICABLES PARA OSTEOPOROSIS EN MUJERES POSMENOPÁUSICAS

\begin{tabular}{|c|c|c|c|c|c|c|c|}
\hline \multirow{3}{*}{$\begin{array}{l}\text { FACTOR } \\
\text { DE RIESGO }\end{array}$} & \multicolumn{4}{|c|}{ DMO } & \multirow{3}{*}{ OR } & \multirow{3}{*}{ IC95\% } & \multirow{3}{*}{ Valor $\mathrm{p}$} \\
\hline & \multicolumn{2}{|c|}{ NORMAL } & \multicolumn{2}{|c|}{ ALTERADA* } & & & \\
\hline & $\mathrm{Fa}$ & $\%$ & $\mathrm{Fa}$ & $\%$ & & & \\
\hline Sobrepeso/Obesidad & & & & & 0,98 & $0,60-1,15$ & 0,957 \\
\hline Presente & 17 & 28,3 & 21 & 35,0 & & & \\
\hline Ausente & 10 & 16,7 & 12 & 20,0 & & & \\
\hline $\begin{array}{l}\text { Suplementación de calcio/ } \\
\text { vitamina D }\end{array}$ & & & & & 1,70 & $1,00-2,80$ & 0,019 \\
\hline Presente & 9 & 15,0 & 21 & 35,0 & & & \\
\hline Ausente & 18 & 30,0 & 12 & 20,0 & & & \\
\hline $\begin{array}{l}\text { Consumo deficitario de } \\
\text { calcio/vitamina D }\end{array}$ & & & & & 1,20 & $0,70-1,90$ & 0,373 \\
\hline Presente & 10 & 16,7 & 16 & 26,7 & & & \\
\hline Ausente & 17 & 28,3 & 17 & 28,3 & & & \\
\hline Hábito tabáquico & & & & & 1,70 & $1,10-2,80$ & 0,013 \\
\hline Presente & 7 & 11,7 & 19 & 31,7 & & & \\
\hline Ausente & 20 & 33,3 & 14 & 23,3 & & & \\
\hline Consumo de alcohol & & & & & 1,20 & $0,20-5,59$ & 0,760 \\
\hline Presente & 4 & 6,7 & 4 & 6,7 & & & \\
\hline Ausente & 23 & 38,3 & 29 & 48,3 & & & \\
\hline Consumo de café & & & & & 3,20 & $1,40-7,10$ & 0,000 \\
\hline Presente & 10 & 16,7 & 28 & 46,7 & & & \\
\hline Ausente & 17 & 28,3 & 5 & 8,30 & & & \\
\hline Consumo de gaseosa & & & & & 2,50 & $1,18-5,60$ & 0,002 \\
\hline Presente & 13 & 21,7 & 28 & 46,7 & & & \\
\hline Ausente & 14 & 23,3 & 5 & 8,30 & & & \\
\hline Sedentarismo & & & & & 1,80 & $0,70-4,10$ & 0,113 \\
\hline Presente & 19 & 31,7 & 29 & 48,30 & & & \\
\hline Ausente & 8 & 13,3 & 4 & 6,70 & & & \\
\hline Ejercicio físico intenso & & & & & 0,92 & $0,05-15,57$ & 0,953 \\
\hline Presente & 1 & 1,7 & 1 & 1,7 & & & \\
\hline Ausente & 25 & 41,6 & 23 & 53,3 & & & \\
\hline Tendencia a caerse & & & & & 1,23 & $0,07-20,64$ & 0,885 \\
\hline Presente & 1 & 1,7 & 1 & 1,7 & & & \\
\hline Ausente & 26 & 43,3 & 32 & 53,3 & & & \\
\hline Dieta hipoproteica & & & & & 1,30 & $0,80-2,20$ & 0,243 \\
\hline Presente & 14 & 23,3 & 22 & 36,7 & & & \\
\hline Ausente & 13 & 21,7 & 11 & 18,3 & & & \\
\hline Dieta vegetariana & & & & & 0,59 & $0,05-6,95$ & 0,676 \\
\hline Presente & 1 & 1,7 & 2 & 3,3 & & & \\
\hline Ausente & 26 & 43,3 & 31 & 51,7 & & & \\
\hline
\end{tabular}

* Osteopenia-Osteoporosis 
Diversos estudios han evidenciado una clara relación entre la producción ovárica de estrógenos y DMO, de tal manera que pacientes con ooforectomía antes de la edad esperada para una menopausia natural presentan un riesgo incrementado de osteoporosis $(17,29,30,36,37)$. Sin embargo, otras investigaciones no han encontraron diferencias significativas ni en la DMO (38) ni en las tasas de fracturas (39) al comparar el tipo de menopausia, por lo que consideraban que no habían efectos a largo plazo de la histerectomía y la ooforectomía bilateral sobre la pérdida ósea.

Diversos estudios han señalado que el uso de la terapia hormonal, en sus diversas formulaciones, como un factor protector para la prevención de la osteoporosis puesto que aumenta la densidad mineral ósea porque actúa directamente en los receptores estrogénicos del hueso y el efecto de la disminución del riesgo de fractura cuando se administra en la etapa posmenopáusica (20,32,40-47). Sin embargo, el uso del tratamiento hormonal no fue considerado en el presente estudio, siendo excluidas las pacientes que utilizaban esta terapéutica, a modo de evitar su influencia en los resultados y poder determinar con mayor precisión la relación o no de las alteraciones en la DMO.

Son múltiples los factores de riesgos modificables asociados a la osteoporosis $(1,6,10,20,32,48)$. En esta serie, los principales resultaron ser el consumo excesivo de café (OR [IC95\%]= 3,2 [1,4-7,1]; $\mathrm{p}<0,05) \mathrm{o}$ de gaseosas (OR $[\mathrm{IC} 95 \%]=2,5[1,18-$ $5,6] ; p<0,05)$. Al respecto, algunos estudios han considerado que una ingesta diaria superior a 300 $\mathrm{mg} /$ día (2 tazas) de cafeína acelera la pérdida de masa ósea en mujeres posmenopáusicas y aumenta el riesgo de fracturas osteoporóticas $(25,28)$; asimismo, el consumo excesivo de gaseosas, las cuales son ricas en fosfatos, se han relacionado con una alta incidencia de osteoporosis y fracturas osteoporóticas, puesto que éstos fosfatos fijan el calcio y evitan su absorción $(6,26)$. En esta serie el consumo de gaseosas demostró tener un mayor riesgo que el atribuido en otra investigación (28), donde si bien se ha mostrado significancia estadística la asociación ha sido débil $(\mathrm{OR}=1,4 ; p<0,05)$.

De igual manera, aunque con una asociación débil pero significativa se identificaron otros factores de riesgo como el hábito tabáquico (OR [IC95\%]= $1,7[1,1-2,8] ; p<0,05)$ y la falta de suplementación de calcio más vitamina D (OR [IC95\%]= 1,7 [1,0$2,8] ; p<0,05)$. El tabaquismo se ha asociado con la disminución de la DMO y el riesgo aumentado de fracturas (28), además de ser más recurrentes y precisar más tiempo para su curación (23); se cree que el tabaco pueda tener un efecto tóxico directo, disminuyendo la actividad osteoblástica del hueso y de la disminución de la absorción intestinal de calcio, además de tener un efecto antiestrogénico $(24,47)$.
Por otra parte, se sabe que la suplementación con calcio durante la posmenopausia retarda la pérdida de masa ósea y por ende el inicio de la osteoporosis (22), por lo que se recomienda la ingesta de al menos 1500 mg/día para conseguir un balance metabólico equilibrado (21). De igual forma, debe suplementarse vitamina D (400U/día) y la exposición a la luz solar puesto que la disminución de los niveles séricos de 25 - hidroxi-vitamina D está asociado con un elevado riesgo de fractura de cadera en mujeres mayores de 65 años (10).

A diferencias de otras investigaciones, factores que tradicionalmente han sido asociados con el riesgo de presentar osteoporosis como ser de descendencia caucásica $(9,10)$, la edad $(8)$, menopausia prematura $(14,15,26,30)$, evolución de la menopausia mayor a 10 años (19), el antecedentes de fracturas atraumáticas (3), sobrepeso u obesidad (32), abuso de alcohol (22), el sedentarismo $(19,25)$, mujeres con tendencia a caerse (24), regímenes nutricionales vegetarianos o con exceso de proteínas (6), no demostraron diferencias causales en el presente estudio.

Una fortaleza del estudio efectuado es que la mayor parte de la información disponible sobre factores de riesgo proviene de países desarrollados, no siendo los mismos directamente aplicables a otras poblaciones, y menos a las de países en desarrollo como es el caso de Venezuela; por tanto, resulta un aporte significativo evidenciar la elevada presencia en la muestra estudiada de algunos de estos factores, siendo posible modificar muchos de ellos. Asimismo, la ausencia de suplementación con calcio y vitamina $D$, aunada a la alta frecuencia de sobrepeso u obesidad, habito caféico acentuado y elevado consumo de gaseosas, pone en evidencia la presencia de malos hábitos nutricionales en estas mujeres, lo cual infiere que el aporte de estos nutrientes en la dieta también debe ser insuficiente; además de que también implican riesgo para presentar enfermedad cardiovascular o metabólica.

Si bien el tipo y diseño de la investigación presenta algunas limitaciones para efectuar un análisis de causalidad directa y no excluye otros efectos del proceso natural de envejecimiento que pueden influir en la calidad del hueso, los resultados del trabajo informa el evidente riesgo que presentan estas mujeres posmenopáusicas para desarrollar osteoporosis. De igual forma, el estudio tiene limitaciones para que los resultados sean generalizables, puesto que se realizó un muestreo intencionado, por tanto, sería recomendable continuar el mismo, incluyendo una mayor cantidad de mujeres posmenopáusicas seleccionadas mediantes criterios más rigurosos y en donde se pudiesen incluir otras variables que pudiesen tener algún efecto sobre la DMO. 


\section{CONCLUSIÓN}

Existe una alta prevalencia de alteraciones en la DMO en las mujeres posmenopáusicas evaluadas en la Maternidad "Dr. Armando Castillo Plaza", de Maracaibo, Venezuela, siendo los principales factores de riesgo determinados la presencia de menopausia quirúrgica, el consumo de gaseosas o café, el tabaquismo y la ausencia de suplementación con calcio y vitamina $D$. Se recomienda realizar la densitometría ósea (DEXA) en la mujer posmenopáusica o que presenten factores de riesgo, de manera de poder realizar diagnósticos y tratamientos oportunos que permitan reducir las secuelas de las fracturas osteoporóticas y mejorar la calidad de vida de las mujeres posmenopáusicas.

\section{REFERENCIAS}

1. Reyes J, Moreno J. Prevalencia de osteopenia y osteoporosis en mujeres posmenopáusicas. Aten Primaria 2005;35(7):342-7.

2. Fernández DG, Mora C, Reyes E, Londoño JD, Santos $\mathrm{P}$, Valle-Oñate $\mathrm{R}$. Tratamiento farmacológico de la osteoporosis postmenopáusica. Rev Colomb Reumatol 2010;17(2):96-110.

3. Anil G, Guglielmi G, Peh WCG. Radiology of Osteoporosis. Radiol Clin N Am 2010;48:497-518.

4. U.S. Department of Health and Human Services. Bone health and osteoporosis: a report of the surgeon general. 2004. [Documento en línea]. Disponible en: http://www.surgeongeneral.gov/library/bonehealth/ content.html. Fecha de consulta: 27 de Abril de 2012.

5. World Health Organization. Assessment of Fracture Risk and its Application to Screening for Postmenopausal Osteoporosis. WHO Technical Report Series 843. Ginebra: WHO; 1994. p. 26.

6. Terán J, Teppa A, Febres C. Actualidad en el diagnóstico de la osteoporosis posmenopáusica. Rev Obstet Ginecol Venez 2007;67(2):115-25.

7. Masoni A, Morosano M, Florencia M, Pezzotto SM, Sanchez A. Factores de riesgo para osteoporosis y fracturas de cadera. Análisis multivariado. Medicina 2007;67(5):423-8.

8. Vázquez M, López A, Isasi C, Aguado P. Fractura osteoporótica: valoración del riesgo en la práctica clínica. Med Clin (Barc) 2007;129(11):418-23.

9. Simon LS. Osteoporosis. Rheum Dis Clin N Am 2007;33:149-176.

10. González LA, Vásquez GM, Molina JF. Epidemiología de la osteoporosis. Rev Col Reum 2009;16(1):61-75.

11. Compston J. Osteoporosis: Social and Economic Impact. Radiol Clin N Am 2010; 48:477-82.

12. Ferrari S. Human genetics of osteoporosis. Best Pract Res Clin Endocrinol Metab 2008;22(5):723-35.

13. International Menopause Society. Recomendaciones sobre la terapia hormonal posmenopáusica. Revista del Climaterio 2007;10(56):29-33.

14. Francucci CM. Effect of natural early menopause on bone mineral density. Maturitas 2008;59(4):323-8.

15. Gallagher JC. Effect of early menopause on bone mineral density and fractures. Menopause 2007;14(3):567-71.
16. Sioka C. Age at menarche, age at menopause and duration of fertility as risk factors for osteoporosis. Climacteric 2010;13(1):63-71.

17. Balderramo DC, Ramacciotti CF, Douthat WG. Factores de riesgo para osteoporosis primaria en mujeres de Córdoba, Argentina. Medicina (Buenos Aires) 2004;64(5):400-6.

18. Gallo JL. Conservación de los ovarios frente a ooforectomía bilateral en pacientes sometidas a histerectomía por procesos benignos. Clín Inv Ginecol Obstet 2009;36(3):94-8.

19. Buttros DAB, Nahas-Neto J, Nahas EAP, Cangussu LM, Barral ABCR, Kawakami MS. Fatores de risco para osteoporose em mulheres na pós-menopausa do sudeste brasileiro. Rev Bras Ginecol Obstet 2011;33(6):295-302.

20. Özdemir F, Demirbağ D, Türe M. Do Dietary Calcium Intake and Hormone Replacement Therapy Affect Bone Mineral Density in Women?. Trakya Univ Tip Fak Derg 2008;25(2):105-9.

21. González J, Guañabens N, Gómez C, del Río L, Muñoz $\mathrm{M}$, Delgado $\mathrm{M}$, et al. Guías de práctica clínica en la osteoporosis posmenopáusica, glucocorticoidea y del varón. Sociedad Española de Investigación Ósea y del Metabolismo Mineral. Rev Clín Esp 2008;208(Supl. 1):1-24.

22. López JE, López-Salazar JE, López Y, Fasanella H. Osteoporosis: alimentación, calcio, vitamina $D$ y ejercicio. Gac Méd Caracas 2007;115(4):286-91.

23. Lewiecki EM. Prevention and treatment of postmenopausal osteoporosis. Obstet Gynecol Clin N Am 2008;35:301-15.

24. Hermoso MT. Clasificación de la osteoporosis. Factores de riesgo. Clínica y diagnóstico diferencial. An Sist Sanit Navar 2003;26(Suppl. 3):29-52.

25. Arana E, Gutiérrez I, Ecenarro A, Asua J. Prevalencia de ciertos hábitos determinantes de osteoporosis en mujeres postmenopáusicas del País Vasco en 2003. Rev Esp Salud Pública 2007;81(6):647-56.

26. Pinheiro MM. Clinical risk factors for osteoporotic fractures in Brazilian women and men: the Brazilian Osteoporosis Study (BRAZOS). Osteoporos Int 2009;20(3):399-408.

27. Rivera MT, Parra MS, Barriguete JA. Trastornos de la conducta alimentaria como factor de riesgo para osteoporosis. Salud Pública Méx 2005;47(4):308-18.

28. Aguilera ML, Rivera JA, Trujillo HM, Ruiz JM, Rodríguez ME. Impacto de los factores de riesgo en osteoporosis sobre la densidad mineral ósea en mujeres perimenopáusicas de la Ciudad de Querétaro, México. Arch Latinoam Nutr 2013;63(1):21-8.

29. Riera G. Epidemiology of osteoporosis in Latin America 2008. Salud Pública Méx 2009;51(Supl. 1):s52-s55.

30. Couto D, Nápoles D, Deulofeu I. Osteoporosis posmenopausia según densitometría ósea. MEDISAN 2011;15(12):1765-74.

31. Pineda LM. Osteoporosis en el climaterio y menopausia. Tesis de especialización. Postgrado de Obstetricia y Ginecología. Facultad de Medicina, Universidad del Zulia. 2008. [Tesis en línea] Disponible en: http://tesis. luz.edu.ve/tde_busca/arquivo.php?codArquivo=2928. Fecha de recuperación: 10 de Mayo de 2014.

32. Rosales E, Muñoz JM, Arias R. Prevalencia de osteopenia y osteoporosis en mujeres posmenopáusicas y su relación con factores de riesgo. Ginecol Obstet Mex 2014;82(4):223-8. 
33. De Lago AA, Parada TM, Somera IJ. Prevalencia de osteoporosis en población abierta de la Ciudad de México. Ginecol Obstet Méx 2008;76:261-6.

34. Bahar S. Comparison of the acute alterations in serum bone turnover markers and bone mineral density among women with surgical menopause. Eur J Obstet Gynecol Reprod Biol 2011;159(1):194-7.

35. Yoshida T, Takahashi K, Yamatani H, Takata K, Kurachi H. Impact of surgical menopause on lipid and bone metabolism. Climacteric 2011;14(4):445-52.

36. Özdemir S. Compared effects of surgical and natural menopause on climacteric symptoms, osteoporosis, and metabolic syndrome. Int J Gynaecol Obstet 2009;10(1):57-61.

37. Özkaya E, Cakir E, Okuyan E, Cakir C, Ustün G, Küçüközkan T. Comparison of the effects of surgical and natural menopause on carotid intima media thickness, osteoporosis, and homocysteine levels. Menopause 2011;18(1):73-6.

38. Kritz-Silverstein D, von Mühlen DG, Barrett-Connor E. Hysterectomy and oophorectomy are unrelated to bone loss in older women. Maturitas 2004;47(1):61-9.

39. Vesco KK, Marshall LM, Nelson HD, Humphrey L, Rizzo J, Pedula KL, et al. Surgical menopause and nonvertebral fracture risk among older US women. Menopause 2012;19(5):510-6.

40. Rossouw JE, Anderson GL, Prentice RL, LaCroix AZ, Kooperberg C, Stefanick ML, et al. Risks and benefits of estrogen plus progestin in healthy postmenopausal women. JAMA 2002;288(3):321-33.

41. Rivera MVC, Halabe ChJ. Actualidades en terapia hormonal estrogénica y osteoporosis posmenopáusica. Med Int Mex 2005;21:431-9
42. Britto R, Araújo L, Barbosa I, Silva L, Rocha S, Valente AP. Hormonal therapy with estradiol and testosterone implants: bone protection?. Gynecol Endocrinol 2011;27(2):96-100.

43. Pasco JA, Kotowicz MA, Henry MJ, Sanders KM, Seeman E, Nicholson GC. Hormone therapy and risk of non-vertebral fracture: Geelong Osteoporosis Study. Osteoporos Int.2004;15(6):434-8.

44. North American Menopause Society. Recommendations for estrogen and progestogen use in peri and postmenopausal women: October 2004 position statement of The North American Menopause Society. Menopause 2004;11:589-600.

45. Palacios S. Advances in hormone replacement therapy: making the menopause manageable. BMC Women's Health. 2008; 8 (22). [Periódico en línea]. Disponible en: http://www.biomedcentral.com/14726874/8/22. Fecha de recuperación: 7 de Mayo de 2014.

46. Rincón O, Díaz I, Pérez LE. Patogénesis de la osteoporosis: papel de los estrógenos. Rev Colomb Obstet Ginecol 2007;58(2):142-50.

47. Landa MC. Papel de la terapia hormonal sustitutiva, en la prevención y tratamiento de la osteoporosis menopáusica. Anales Sis San Navarra 2003;26(Suppl. 3):99-105.

48. Mendoza MÁ, Ramírez MC, Velasco JF, Rivera JG; Nieva RN, Valdez LÁ. Paridad y menarquía como factores de riesgo para osteoporosis en mujeres posmenopáusicas. Ginecol Obstet Mex 2014;82(2):75-82. 